\title{
Kernos
}

Revue internationale et pluridisciplinaire de religion grecque antique

$22 \mid 2009$

Varia

\section{Angelo Brelich, Il politeismo}

\section{Gabriella Pironti}

\section{CpenEdition \\ Journals}

\section{Édition électronique}

URL : http://journals.openedition.org/kernos/1793

DOI : 10.4000/kernos. 1793

ISSN : 2034-7871

\section{Éditeur}

Centre international d'étude de la religion grecque antique

\section{Édition imprimée}

Date de publication : 1 janvier 2009

Pagination : 312-314

ISSN : 0776-3824

\section{Référence électronique}

Gabriella Pironti, «Angelo Brelich, II politeismo », Kernos [En ligne], 22 | 2009, mis en ligne le 15

septembre 2011, consulté le 21 septembre 2020. URL : http://journals.openedition.org/kernos/1793 ;

DOl : https://doi.org/10.4000/kernos. 1793

Ce document a été généré automatiquement le 21 septembre 2020.

Kernos 


\title{
Angelo Brelich, Il politeismo
}

\author{
Gabriella Pironti
}

\section{RÉFÉRENCE}

Angelo BRELICH, Il politeismo, a cura di Marcello Massenzio e Andrea Alessandri, prefazione di Marc Augé, Roma, Editori Riuniti, 2007. 1 vol. 15 落 $21 \mathrm{~cm}, 143$ p. (Opere di Brelich). ISBN : 978-88-359-5986-1.

1 On ne peut que saluer la publication, par les soins de M. Massenzio et A. Alessandri, de la version rédigée du cours donné par Angelo Brelich entre 1957 et 1958 à l'Université de Rome. Ce petit volume est d'autant plus précieux que non seulement il constitue l'occasion de mieux connaître l'œuvre du célèbre historien des religions italien, mais il permet également au lecteur de prendre place dans sa salle de cours et d'en " écouter » presque les réflexions sur le polythéisme, sujet primordial mais peu exploré à l'époque. Ce qui apparaît comme novateur, par rapport aux historiens des religions qui l'ont précédé, est surtout le questionnement choisi par Brelich, qui essaie d'identifier la spécificité de la forme religieuse dénommée "polythéisme ", sans la subordonner à la question du monothéisme, et interroge de près la notion de divinité ainsi que la manière dont les divinités au pluriel se structurent dans un panthéon.

Dès le premier chapitre, consacré au problème de la formation du polythéisme, Brelich prend ses distances par rapport aux tenants de l'évolutionnisme qui, comme E.B. Tylor, avaient réduit le polythéisme à une simple étape sur le chemin qui, de l'animisme des origines, aurait abouti au monothéisme. Dans le sillage de son maître Raffaele Pettazzoni, il critique tout aussi âprement la thèse du monothéisme primordial, déchu en polythéisme, comme le voulait Wilhelm Schmidt, et revendique la nécessité d'étudier le polythéisme pour lui-même afin d'évaluer correctement les circonstances historiques de ses origines : « senza conoscere, infatti, il fenomeno stesso, è impossibile interpretarlo storicamente » (p. 24). Pour établir les traits caractéristiques du polythéisme en général, Brelich se sert d'un comparatisme tous azimuts et convoque, à côté des religions de l'Antiquité, les polythéismes modernes d'Asie et d'Afrique. Les 
deux éléments fondamentaux où il reconnait la spécificité de cette typologie religieuse sont, d'un côté, la notion de divinité, trait essentiel que les poly-théismes auraient en commun avec les mono-théismes, et de l'autre, la pluralité organisée des dieux individuels, trait qui en revanche constituerait la différence essentielle entre les premiers et les seconds. Il distingue nettement la divinité, theos (être personnel non humain, ayant une large sphère d'action, actif dans le monde et objet d'un culte) des autres êtres mythiques mais non divins, ancêtres, esprits, etc., aboutissant ainsi à établir une différence de fond entre le polythéisme et les religions "pré-déistiques ", qui ne posséderaient pas ou pas encore l'idée de dieu. Il finit par nier, sur cette base, tout caractère primitif au polythéisme, qui lui apparaît en revanche comme l'expression de civilisations ayant atteint un haut niveau de complexité, dans la stratification sociale comme dans la spécialisation des métiers. En revenant, dans la partie conclusive, sur le débat autour de la genèse du polythéisme, Brelich avance des critiques à la thèse diffusionniste qui faisait de la Mésopotamie le berceau de cette typologie religieuse, mais admet la possibilité que les contacts entre différentes civilisations supérieures, notamment dans l'aire méditerranéenne, aient pu contribuer à développer la religion de certaines d'entre elles en direction d'un polythéisme plus affirmé.

3 Il n'est pas le cas d'insister ici sur les contradictions qui parcourent l'exposé de Brelich : en effet, malgré lui, l'historien des religions n'arrive pas à se défaire complètement d'une pensée évolutionniste qui était sans doute dans l'air du temps. En outre, tout attentif qu'il soit à ne pas dissocier la visée comparatiste du respect pour les différentes contextes historiques mis en regard, il reste toutefois dépendant de sa volonté d'identifier des traits communs pour aboutir à une théorie générale du polythéisme, qui se situe de fait en deçà de l'histoire. Mais il ne faut pas oublier non plus que le schématisme de certains arguments est sans doute lié, dans ce cadre précis, à la finalité pédagogique de la démonstration.

D'autre part, l'effort théorique qui caractérise la réflexion de Brelich a le mérite indiscutable de porter l'attention sur le problème, toujours actuel, de la représentation du divin dans un système polythéiste, qu'il s'agisse du rapport entre une divinité et ses fonctions ou bien des structures internes des panthéons. En s'inscrivant contre l'interprétation qui voyait dans les dieux de simples projections des éléments naturels, Brelich perçoit clairement la complexité des divinités du polythéisme, qui tissent des relations étroites entre plusieurs éléments et niveaux de la réalité, y compris la nature. À travers le cas du dieu védique Agni, il montre bien que l'ensemble des aspects correspondant à chaque divinité est indissociable d'un contexte historique et culturel donné, où seulement cet ensemble peut retrouver tout son sens, révélant ainsi son organicité interne. Brelich conçoit le panthéon comme une sorte de projection, sur le plan divin, de la manière dont une société se structure, interprète le monde et crée un rapport culturellement réglé avec les aspects « incontrôlables » de la réalité. Le monde divin, tout comme chaque divinité personnelle, apparaît dans cette perspective comme un ensemble organique et cohérent.

5 Brelich s'intéresse moins au fonctionnement du polythéisme qu'au processus qui voit cette forme de religion se traduire en réalité selon ses «tendances» propres et en s'affirmant contre d'autres "tendances", par exemple celles qui appartiennent aux religions «pré-déistiques». Les tensions qu'il reconnaît dans le polythéisme visent avant tout à la formation des figures divines: la tension vers l'anthropomorphisme, 
contre le thériomorphisme et l'aniconisme, celle qui aboutit à la cristallisation du monde divin en un nombre limité de dieux, contre la prolifération des divinités mineures, celle qui va vers une différentiation nette des profils des dieux et une spécialisation de leurs prérogatives. Ces tendances seraient fonctionnelles à la constitution de divinités bien définies, douées chacune d'une personnalité propre, dont les sphères d'action respectives se délimiteraient nettement les unes par rapport aux autres. La volonté de montrer le polythéisme in fieri aboutit donc chez Brelich à l'image d'un panthéon statique où chaque divinité se définirait par rapport à la case qu'elle vient à occuper à l'issue d'un processus d'individuation, concentration et différentiation. "Ad un antico greco - dit-il - non sarebbe mai venuto in mente di chiedere guarigione o prole ad Ares, né una vittoria in guerra ad Afrodite» (p. 56). Il est toujours risqué d'avoir recours aux « jamais » et aux « toujours » en histoire, et l'étude du polythéisme hellénique ne fait pas exception à cette règle de prudence: en l'occurrence, il est bien des cas où des Grecs ont demandé la victoire en guerre à Aphrodite et, de manière plus générale, de nouvelles données et de récentes analyses sont venues démontrer depuis lors le caractère anachronique du portrait canonique de la «déesse de l'amour». Mais pour en arriver là, il a fallu le long parcours qui, des études de Georges Dumézil, avec l'introduction de la notion de mode d'action, à celles de Jean-Pierre Vernant, avec le passage de la notion de "personne " à celle de puissance divine, et aux critiques formulées par Marcel Detienne, a conduit à remettre en question tout modèle statique du panthéon grec et à repenser les divinités et les agencements panthéoniques en fonction de leur capacité de se décliner et de se "réagencer » suivant les différents contextes.

6 Mais si certains des points de départ et des conclusions de Brelich ne sont plus d'actualité, les réflexions qu'il proposait à ses étudiants sur plusieurs aspects des religions antiques gardent aujourd'hui encore un grand intérêt. S'interrogeant sur les moyens que le polythéisme mettrait en œuvre pour réaliser ses «tendances », il passe en revue le fonctionnement des théonymes et des épiclèses, celui de la représentation anthropomorphe, et donc "genrée ", des dieux, et celui de la généalogie, en tant qu'outil fondamental pour définir la place d'un dieu dans le monde divin. Même les aspects les plus significatifs de la vie cultuelle lui apparaissent avant tout comme des moyens voués à définir la personnalité divine et à différencier entre elles les divinités du polythéisme : les différents procédés sacrificiels, la topographie "sacrée », à savoir le choix du lieu de culte et les particularités de sa structure, la place de chaque fête dans le calendrier, avec son déroulement et sa temporalité spécifiques, sans oublier le personnel cultuel, dont les caractères requis auraient également une fonction connotative. Certes, en approchant les faits cultuels exclusivement sous l'angle de la "tendance", Brelich finit par subordonner au processus de différentiation cette volonté de communication avec les dieux qui est essentielle pour comprendre le sens des rituels dans la vie quotidienne des pratiquants. D'autre part, il a le mérite d'attirer l'attention sur un élément fondamental, mais souvent négligé, à savoir le fait que les pratiques rituelles relèvent pleinement de la représentation polythéiste du divin et qu'elles peuvent aussi avoir une charge connotative. Il reste, cependant, que, dans la perspective de ceux qui accomplissent les gestes rituels, cette «différentiation » est moins la fin d'un processus que le présupposé d'une adresse rituelle différenciée, capable d'identifier la puissance divine adéquate au contexte et d'établir avec elle une communication efficace. 
7 La préface de Marc Augé signale de manière synthétique les mérites et les limites de ce volume qui contient aussi, en plus d'une postface des éditeurs et d'une note biobibliographique de l'auteur, l'important article « Der Polytheismus » (1960), republié ici dans sa traduction italienne, où Brelich revient sur les principaux arguments de son analyse du polythéisme. Ce petit livre est une lecture obligée pour tous ceux qui s'intéressent de près ou de loin à l'histoire des religions, en général, et à celle des polythéismes antiques, en particulier. Non seulement en vertu de la place d'honneur qui revient à Brelich dans l'histoire des études, mais aussi parce que cette publication nous invite tous à réfléchir de manière plus approfondie sur le rapport entre représentation du divin et fonctionnement du polythéisme.

\section{AUTEURS}

\section{GABRIELLA PIRONTI}

Université de Naples 